koordiniert werden könnten. Auch zeige sich, daß in Südafrika in weit höherem Maße die Bevölkerung in den Aufarbeitungsprozeß integriert sei, während in Deutschland die staatliche Initiative dominiere. Dies hänge damit zusammen, daß dieser Prozeß in Deutschland eher als ein juristischer, denn als gesellschaftspolitischer Prozeß gesehen werde. Auffällig sei darüber hinaus die vorrangige Stellung der Wahrheitsfindung in Südafrika, für die letztendlich die Sanktionierung des begangenen Unrechts "geopfert" würde (S. 206). In Deutschland hingegen bestehe keine derartige Gewichtung, sondern beide Elemente bildeten zwei unterschiedliche und unabhängige Elemente der Aufarbeitung.

In ihrem kurzen Ausblick auf die Eignung der TRC zur Förderung der Versöhnung kommt die Autorin zu dem Ergebnis, daß "die TRC durch ihren wertvollen Beitrag zur Aufdeckung der Wahrheit in jedem Fall eine überragend wichtige Grundvoraussetzung für die Versöhnung des südafrikanischen Volkes schafft" (S. 211). Die Versöhnung könne nicht allein durch die Arbeit der TRC herbeigeführt werden, sondern sei ein langwieriger gesellschaftlicher Prozeß, der selber "wachsen" müsse.

Das vorliegende Werk bietet eine wertvolle Zusammenfassung der Konzeption, Aufgabengebiete und Arbeitsweisen der TRC. Verbunden mit den Hintergrundinformationen zur Apartheidpolitik und den Wahrheitskommissionen in Lateinamerika, die wesentlichen Einfluß auf die südafrikanische Wahrheitskommission hatten, stellt sich die Dissertation als wichtige Informationsquelle bei der Bewertung des inzwischen am 29. Oktober 1998 erschienenen Abschlußberichts der südaf rikanischen Wahrheits- und Versöhnungskommission dar.

Peggy Wittke

\title{
Stefanie Pautke
}

Die kartellrechtliche Erfassung konglomerater Konzentration in der Republik

\section{Südaf rika}

Schriftenreihe Recht und Verfassung in Südafrika, Band 2 (Herausgegeben von Ulrich

Karpen, Ingo von Münch und Hans-Peter Schneider)

Nomos Verlagsgesellschaft, Baden-Baden, 1998, 208 S., DM 66,--

Nicht nur die demokratischen Strukturen am Kap der Guten Hoffnung unterliegen einem mühsamen (noch nicht abgeschlossenen) Entwicklungsprozeß; auch die Wirtschaftsverfassung Südafrikas muß langsam ihren Sonderweg verlassen und zu vergleichbaren Formen moderner Volkswirtschaften aufschließen. Die speziellen Umstände der Besiedlung, der politischen Herrschaft und der Industrialisierung haben dort ebenso tiefe Spuren gegraben wie die Zeit der Apartheid, in der sich westliche Investoren aus ihren Engagements zurückzogen. Das Diktat des Überlebens diktierte damals auch die Entstehung oligopoler Machtgebilde in der Wirtschaft. 
Diese Zeit ist nun vorbei. Die Reformer erwartet ein reiches, zum Teil singuläres Feld an Aufgaben mit vielfältigen - auch delikaten - Ansätzen. Nicht zu Unrecht hat die Verfasserin mit ihrer Arbeit, die als juristische Dissertation von der Universität Göttingen angenommen worden ist, ihrem Text die Schemata der fünf südafrikanischen Großkonzerne beigefügt: Anglo-American Corporation, Sanlam, Rembrandt-Group, South-AfricanMutual und Liberty-Group zeigen einen feingeknüpften Teppich von Strukturen, die zum Teil ineinander greifen und sie als großen Clan mit unterschiedlichen, aber vor allem auch abgestimmten gleichen Interessen ausweisen.

Ob es sich bei dieser großen "Familie" - und anderen, minder großen Verbindungen von ähnlich strukturierten Gebilden - um eine segensreiche Konstellation handelt, untersucht die Verfasserin über 172 Textseiten hinweg. Die in Südaf rika herrschenden konglomeraten Konzentrationen und ihre rudimentäre Regelung stellt sie den Regelungen in Deutschland, der EG und zum Teil auch in den USA gegenüber. Dabei erfüllt die Verfasserin auch hervorragend das Postulat ihres Untertitels "Bestandsaufnahme und Entwicklung von Lösungsansätzen unter besonderer Berücksichtigung deutscher und europäischer Erfahrungen". Ausgehend von einer Bestandsaufnahme mit der Erkenntnis, daß so gut wie alle größeren Privatunternehmen direkt oder indirekt zu einem der fünf Großkonzerne gehören und allein $56 \%$ des Aktienbesitzes in Südafrika drei Familien zugeordnet werden können (den Oppenheimers, den Ruperts sowie den Gordons), gelangt die Verfasserin sehr schnell zur Frage, wo Vorzüge und Gefahren konglomerater Wirtschaftsmacht liegen.

Insgesamt wägt sie klug Positives und Negatives gegeneinander ab. Sie sieht einerseits den "Pool produktiver Ressourcen" (S. 23) durch Effizienzsteigerungen, Sanierungsmöglichkeiten und die Überwindung von Marktzutrittsschranken. Andererseits gewinnt sie nach Prüfung der negativen Aspekte die Erkenntnis: Die jahrelange Apartheid-Politik und die damit verbundene Isolation von den Weltmärkten haben die südafrikanischen Unternehmen zu einer absoluten Größe heranwachsen lassen, die sich in keinerlei Hinsicht mehr mit Synergieeffekten rechtfertigen läßt. Hier gilt vielmehr das Gegenteil: Fehlender Wettbewerb auf den Märkten und kaum vorhandene Konkurrenz haben zahlreiche südafrikanische Unternehmen zum Teil derart ineffizient werden lassen, daß sie bei Aufgabe staatlicher Schutzmaßnahmen durch den bloßen Zutritt ausländischer Wettbewerber rasch an die Grenze ihrer Überlebensfähigkeit geraten. Im wahrsten Sinne des Wortes treffen wir insoweit auf eine Staatsaffäre.

Die Arbeit dient damit nicht nur der Lösung einer in der inneren Analyse des Landes liegenden Aufgabe. Sie will dazu beitragen, Südafrika als fairen Wettbewerbspartner mit seiner Wirtschaft fit für den Weltmarkt zu machen. Freier Fluß von Waren, Dienstleistungen und Kapital soll auch auf faire und jedermann zugängliche Strukturen treffen. Davon ist das Land heute noch weit entfernt. Trotz rudimentärer Ansätze durch die Schaffung von Kommissionen und Wettbewerbsbehörden (hierzu S. 58 ff.) muß eine Entflechtung stattfinden (S. 120 ff.). Freilich hat diese Entflechtung vor dem besonderen Hintergrund der dortigen Verhältnisse nichts gemein mit nur nominell vergleichbaren Vorgängen in der Bundesrepublik Deutschland und der Europäischen Union (S. $121 \mathrm{ff}$.). Die Verfasserin 
schlägt vielmehr ein ganzes Bündel von Maßnahmen vor, so etwa eine Verbesserung des wettbewerblichen Umfeldes durch Maßnahmen außerhalb kartellrechtlicher Regelungen, etwa gesellschaftsrechtlicher Vorschriften in bezug auf personelle Verflechtungen, Wegfall steuerlicher Anreize bei der Behandlung von Dividenden innerhalb eines Konzernverbandes bis zur Verbesserung bestehender gesetzlicher Maßnahmen zur Kontrolle konglomerater Konzentrationen wie Fusionskontrolle und Mißbrauchsaufsicht.

Einen ganz wesentlichen Akzent legt die Arbeit allerdings auf die Maßnahmen einer neuen südafrikanischen Wettbewerbsgesetzgebung zu Unternehmensentflechtung (S. $145 \mathrm{ff}$.). Ergänzungswünsche sind hinsichtlich der Schaffung einer "Position unangemessener wirtschaftlicher Macht" als zentrales Eingreifkriterium für gesetzliche Rechtsfolgen anzumelden (S. 171), etwa durch einen Katalog oder klare Parameter für die Subsumtion. Eindeutige Zustimmung verdient die Verfasserin mit der Forderung nach der Schaffung eines unabhängigen Verwaltungsorgans, das mit kompetenten Experten besetzt wird und damit zur Bildung einer unabhängigen Kartellbehörde führt.

Die Arbeit sollte in einer Phase der wirtschaftspolitischen Überprüfung und Neuorientierung des Landes als wichtiger Beitrag für Lösungsansätze hinzugezogen werden. Sie bietet ein vorzügliches Beispiel der Schriftenreihe, die sich mit zentralen Institutionen, Fragen und Entwicklungen des südafrikanischen Rechts befaßt und tiefgehende Einblicke nicht nur den Interessierten hierzulande hierüber verschafft. Daß der "Umbau" zwar mit Zielstrebigkeit angepackt werden muß, aber mit Augenmaß für das "Machbare" zu erfolgen hat, versteht sich von selbst. Die "Ungeduld des Herzens" als einziger Motor für Reformen könnte das Land leicht in schwere, schadensträchtige Konflikte stürzen.

Heinz Christian Hafke

\section{Das Türkische Strafgesetzbuch / Türk Ceza Kanunu}

vom 1. März 1926 nach dem Stand vom 31. Oktober 1998

Deutsche Übersetzung und Einführung von Silvia Tellenbach

Sammlung ausländischer Strafgesetzbücher in deutscher Übersetzung, Band 10

(zweisprachige Ausgabe)

edition iuscrim, Freiburg im Breisgau, 1998, 339 S., DM 68,--

Angesichts der engen wirtschaftlichen Verflechtung zwischen Deutschland und der Türkei, der zahlreichen hier lebenden Türken und der ebenfalls zahlreichen jährlich in die Türkei aufbrechenden deutschen Urlauber ist eine Unterrichtungsmöglichkeit über das in der Türkei geltende Straf recht eigentlich unverzichtbar. Die einzige bis 1998 greifbare deutsche Textausgabe stammte aber von 1955 und war aufgrund der seither ergangenen zum Teil 